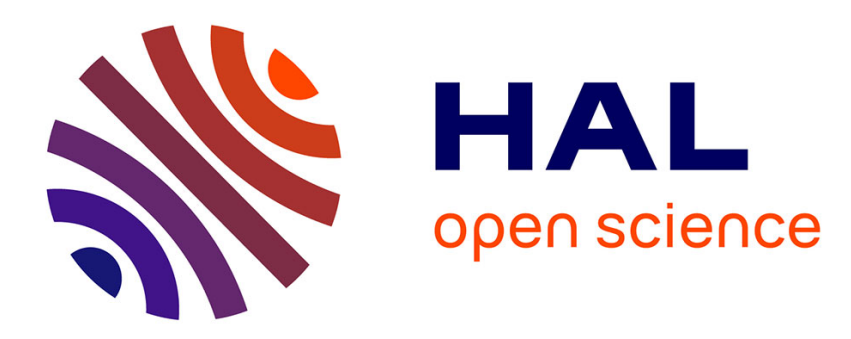

\title{
Absence of Geometrical Regime for Impedance-Type Elastic Scatterers
}

\author{
Ludovic Margerin, Marie Calvet
}

\section{To cite this version:}

Ludovic Margerin, Marie Calvet. Absence of Geometrical Regime for Impedance-Type Elastic Scatterers. Bulletin of the Seismological Society of America, 2018, 10.1785/0120180073 . hal-02324285

\section{HAL Id: hal-02324285 \\ https://hal.science/hal-02324285}

Submitted on 21 Oct 2019

HAL is a multi-disciplinary open access archive for the deposit and dissemination of scientific research documents, whether they are published or not. The documents may come from teaching and research institutions in France or abroad, or from public or private research centers.
L'archive ouverte pluridisciplinaire HAL, est destinée au dépôt et à la diffusion de documents scientifiques de niveau recherche, publiés ou non, émanant des établissements d'enseignement et de recherche français ou étrangers, des laboratoires publics ou privés. 


\title{
Absence of geometrical regime for impedance-type elastic scatterers
}

\author{
L. Margerin and M. Calvet \\ Institut de Recherche en Astrophysique et Planétologie \\ Université Paul Sabatier Toulouse III, C.N.R.S., C.N.E.S. \\ 14 Avenue Edouard Belin, 31400 Toulouse France
}

August 6, 2018

\begin{abstract}
In wave physics, the geometrical limit is defined as a propagation regime where the scattering cross-section $\sigma$ of an object becomes independent of its internal structure and tends to twice its geometrical cross-section $\sigma_{g}$, a result which is particularly well documented in optics. Following the classification of Wu and Aki (1985b), we study the high-frequency scattering limit for velocitytype and impedance-type elastic perturbations. While velocity-type scatterers do follow the geometrical limit $\sigma \rightarrow 2 \sigma_{g}$, the scattering cross-section of impedance-type scatterers depends on both their density and elastic properties at all frequencies. These results are illustrated with the example of a spherical inclusion exhibiting a small contrast of properties with its environment. We derive simple asymptotic formulas that show good agreement with exact solutions of the boundary value problem. Our results confirm the distinct behavior of velocity-type vs impedance type perturbations at all frequencies.
\end{abstract}

\section{Introduction}

In their seminal paper on the scattering of seismic waves by elastic inclusions, Wu and Aki (1985b) introduced the concepts of "velocity-type" and "impedance-type" perturbations. The former (resp. the latter) refers to an object exhibiting a contrast of velocity (resp. impedance) but no contrast of impedance (resp. velocity) with its environment. Wu and Aki (1985b) demonstrated that any general elastic perturbation may be decomposed into a sum of "velocity-type" and "impedancetype" perturbation in a unique way. From a seismological point of view, this decomposition is 
particularly meaningful. Indeed, it may be shown in the long wavelength limit that the radiation pattern of impedance-type perturbations displays large lobes of back-scattering, whereas velocitytype perturbations tend to scatter energy around the forward direction. Note, however, that in isotropic elastic media (and in the long wavelength limit) there is no conversion scattering ( $S$ to $P$ or $P$ to $S$ ) in the exact forward and backward directions due to the symmetry of the elastic tensor. As a consequence of the properties outlined above, one may expect impedance-type perturbations to play an important role in direct wave attenuation and coda wave generation (Sato, 1984; Wu and Aki, 1985a), whereas velocity-type perturbations participate to phenomena associated with forward scattering such as envelope broadening (Sato, 1989).

The separation of elastic fluctuations into velocity-type and impedance-type perturbations is also relevant to the theory of attenuation through discrete disordered media. Kawahara (2011) studied theoretically the case where weak contrast inclusions are dispersed in a homogenous matrix. He compared the classical theory of Foldy (1945) with a continuous approximation where the random medium is described with the aid of a 2-point correlation function. In this approximation, the scatterers are no longer represented as discrete object but rather as fluctuations of a continuum (see Torquato, 2002, for further details). On the one hand, Foldy's theory is based on the exact solution of the scattering problem for a single inclusion and is expected to be valid when the number density of scatterers is low (Foldy, 1945). On the other hand, the equivalent continuum description is expected to be valid for sufficiently weak perturbations only. In the case of spherical inclusions, Kawahara (2011) calculated attenuation using the results of Korneev and Johnson (1996) in the framework of Foldy's theory and the Born approximation in the continuum model. The applicability of Born approximation (also called the Rayleigh-Gans approximation in optics) is usually limited to sufficiently low frequency (Van de Hulst, 1981). In the case of velocity-type perturbations Kawahara (2011) indeed found good agreement between Foldy's theory and the continuum approximation at low frequency, and noted the divergence of Born approximation in the high-frequency limit. In the case of impedance-type perturbations, however, the author pointed out that the two theories agree at all frequencies. He attributed the success of Born approximation to the absence of phase-shift inside the scatterers in the case of pure impedance-type perturbations.

Calvet and Margerin (2018) considered the related problem of a symmetric two-phase elastic random medium. When the volume fractions are equal, the term "symmetric" implies that the 


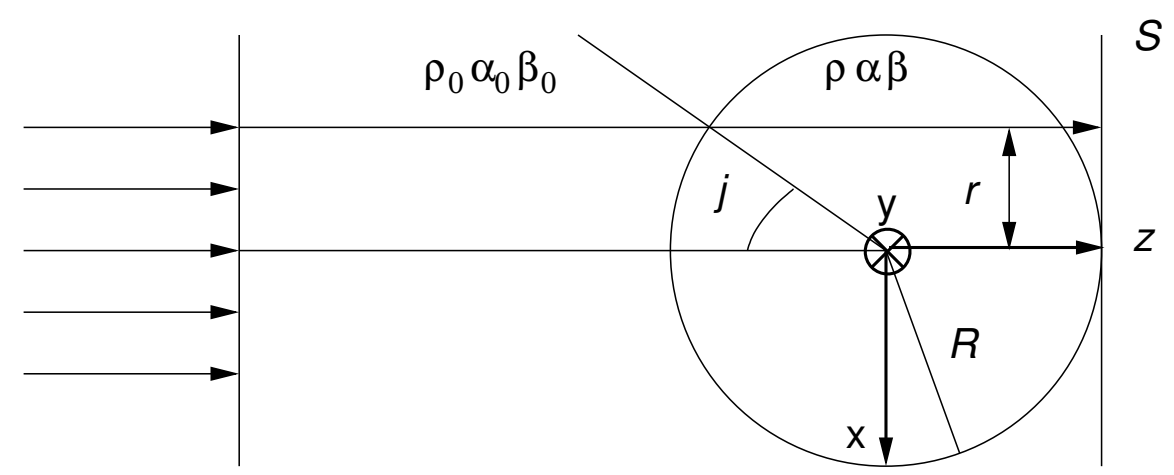

Figure 1: Position of the problem. We consider a plane elastic ( $P$ or $S$ ) wave incident on a sphere of radius $R$. The incidence angle of a given ray is denoted by $j$. The subscript 0 refers to the properties of the matrix with $\rho$ the density, $\alpha$ the $P$-wave velocity and $\beta$ the $S$-wave velocity.

physical properties of the medium are invariant upon inversion of the phases (Torquato, 2002). These authors adopted a continuum description and computed the dispersion and attenuation of elastic waves using a spectral function technique. In this approach, the propagating modes are characterized by measuring both the location of the maximum and the width of the imaginary part of Green's function in the frequency-wavenumber domain. This method is thought to be valid for sufficiently weak perturbations -including in the high-frequency limit-. Similar to the finding of Kawahara (2011), Calvet and Margerin (2018) note that in the case of impedance-type perturbations, calculations of the attenuation based on the Born approximation yield the same result as the spectral function approach (see the erratum to the original article). Furthermore, these authors found that in the high-frequency limit, attenuation does not tend to the usual geometrical limit $l=a$, with $l$ the attenuation length and $a$ the correlation distance of the medium. Note that in the geometrical limit, attenuation is totally independent of the internal properties of the scatterers, a result which is well documented in optics (Van de Hulst, 1981). In sharp contrast, Calvet and Margerin (2018) found on the basis of Born approximation that attenuation by impedance-type perturbations depends on the contrast of elastic properties in the 2-phase medium at all frequencies. This note can be considered as an outlook on the two papers just discussed.

\section{Theory and numerical results}

In order to confirm or infirm the conclusions of Kawahara (2011) and Calvet and Margerin (2018), we reexamine the problem of high-frequency scattering by spherical inclusions (see Figure 1). Our 

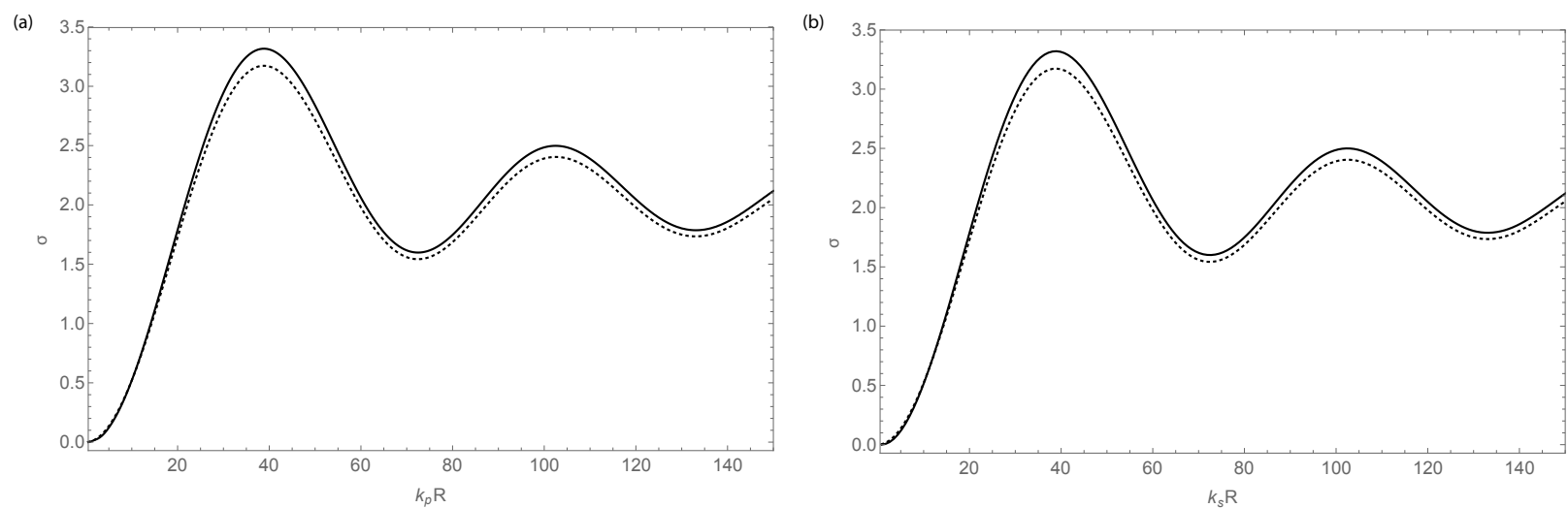

Figure 2: Scattering cross-section (normalized by the geometric cross-section) of $P$ waves (a) and $S$ waves (b) as a function of non-dimensional frequency $k_{p, s} a$ for a velocity-type perturbation equal to $-5 \%$. Solid line: series solution of the BVP, dashed line: Ray theory.

objective is 2-fold: evaluate the validity of Born approximation for impedance-type perturbations and provide an independent confirmation of the absence of geometrical regime in this case. To carry out this task, the outcome of three theoretical approaches will be compared: (1) The formal series solution of the boundary value problem (B.V.P.) for spheres as derived by Korneev and Johnson (1996); (2) A high-frequency approximation which couples ray theory with a classical representation theorem for the scattered field; (3) the Born approximation for scattered elastic wavefields. We begin with a brief summary of known results for velocity-type perturbations and proceede with impedance type perturbations next. In numerical applications, we consider the case where the matrix is a Poisson solid.

\section{Velocity-type perturbations}

Although velocity-type perturbations have previously been treated by Korneev and Johnson (1996) using the formal series solution of the BVP, we propose an alternative derivation based on a method developed in optics by Van de Hulst (1981). In fact, the approach bears many similarities with the screen method which has been widely used in seismology (e.g. Stoffa et al., 1990; Wu, 1994). It is equally applicable to velocity-type and impedance-type perturbations and allows one to understand the physical origin of the difference between the two cases. We show in Figure 2 and 3 the highfrequency behavior of the $P$ and $S$ scattering cross-sections for $-5 \%$ velocity-type and impedancetype perturbations, respectively. The scattering response of the two types of inclusions is strikingly different. In the case of a velocity-type perturbation, the similarity between $P$ and $S$ wave scattering 
behavior is worth noting and can be explained with the aid of ray theory (which is shown by dashed lines in Figures 2 and 3). To obtain an asymptotic high-frequency approximation, we employ the method of Van de Hulst (1981) which consists in computing the forward scattered field using a combination of Huygens principle and ray theory. The main steps of the method are outlined hereafter.

Our starting point is a representation theorem for scattered elastic wavefields (Pao and Varatharajulu, 1976):

$$
\mathbf{u}^{s c}(\mathbf{x})=\int_{S}\left(\mathbf{u}^{s c}\left(\mathbf{x}^{\prime}\right) \cdot\left(\mathbf{n}^{\prime} \cdot \mathbf{\Sigma}^{0}\left(\mathbf{x}, \mathbf{x}^{\prime}\right)\right)-t^{s c}\left(\mathbf{x}^{\prime}\right) \cdot \mathbf{G}^{0}\left(\mathbf{x}, \mathbf{x}^{\prime}\right)\right) d S^{\prime}
$$

For clarity, we recall the physical quantities appearing in Eq. (1). $\mathbf{u}^{s c}$ : the scattered wavefield, $\mathbf{t}^{s c}$ the scattered traction field, $\boldsymbol{\Sigma}^{0}\left(\mathbf{x}, \mathbf{x}^{\prime}\right)$ : stress Green tensor in the matrix for source at $\mathbf{x}^{\prime}$ and receiver at $\mathbf{x}, \mathbf{G}^{0}\left(\mathbf{x}, \mathbf{x}^{\prime}\right)$ : displacement Green tensor in the matrix, $S$ : surface enclosing the scatterer, $\mathbf{n}^{\prime}$ : inward normal to $S$. For our problem, it is convenient to let $S$ coincide with the plane tangent to the sphere at the exit point of the central ray (see Figure 1). To close this surface, we may add a large hemisphere of radius $r$ and let $r \rightarrow \infty$. However, there will be no contribution from this surface thanks to the radiation conditions obeyed by the tensors $\boldsymbol{\Sigma}^{0}$ and $\mathbf{G}^{0}$. Our next step is to approximate the scattered wavefield $\mathbf{u}^{s c}$ on the exit plane $S$. We consider the scattered $P$ waves first. For sufficiently weak perturbations, we may neglect the bending of the rays at the interface between the scatterer and the matrix. In this approximation, the main effect of the scatterer on the total transmitted $P$-wavefield $\mathbf{u}_{p}$ is to modify its phase according to:

$$
\mathbf{u}_{p}(r, z)=\hat{\mathbf{z}} e^{i \omega z / \alpha_{0}+i \delta \phi(r)} \quad z \geq R
$$

where $\omega$ denotes the circular frequency, $\delta \phi(r)$ is the phase-shift induced by the presence of the scatterer, $\hat{\mathbf{z}}$ is the propagation direction (and polarization vector) of the incident $P$ waves, $R$ is the radius of the sphere and $r$ is the transverse distance to the central ray and $\alpha_{0}$ is the wavespeed in the background medium. For simplicity, we set up an $(x, y, z)$ cartesian coordinate system whose origin coincides with the sphere center. From Figure 1, we find $\delta \phi(r)=2 \omega\left(1 / \alpha-1 / \alpha_{0}\right) R \cos j$, where $j$ is the incidence angle of the rays hitting the sphere at a transverse distance $r=\sqrt{x^{2}+y^{2}}=R \sin j$. Using the relation $\mathbf{u}_{p}=\mathbf{u}_{p}^{s}+\mathbf{u}_{p}^{i}$ (the superscript ${ }^{i}$ stands for the incident field), we conclude that 

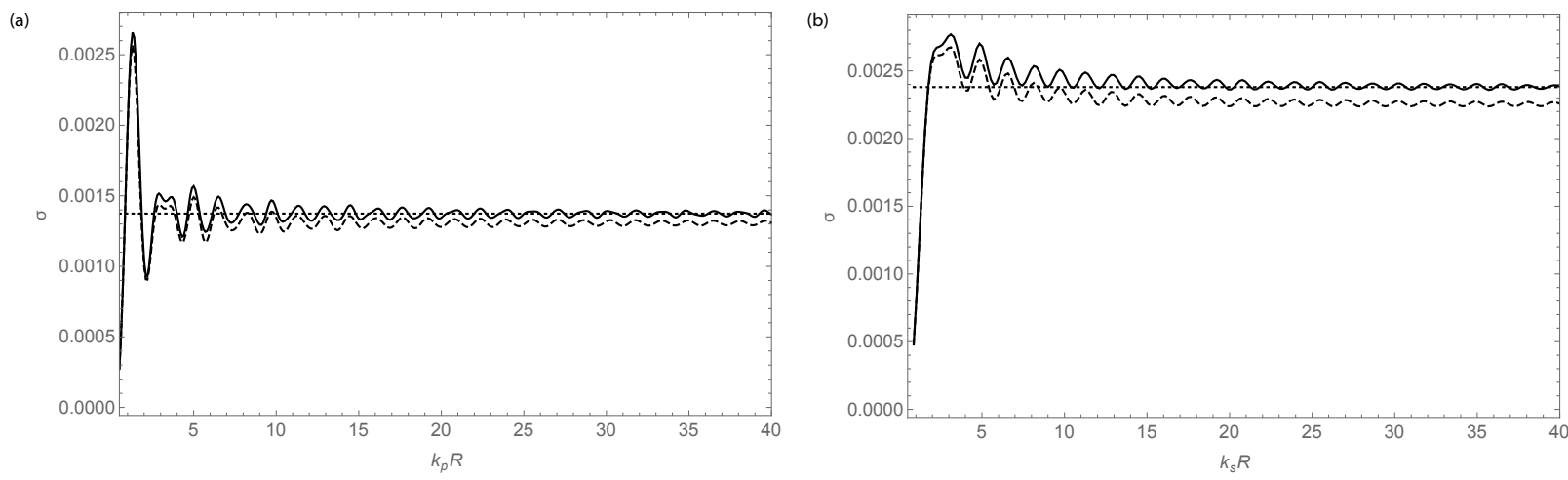

Figure 3: Scattering cross-section (normalized by the geometric cross-section) of $P$ waves (a) and $S$ waves (b) as a function of non-dimensional frequency $k_{p, s} a$ for an impedance type perturbation equal to $-5 \%$. Solid line: series solution of the BVP, dashed line: Born (Rayleigh-Gans) approximation, dotted line: ray theory.

right after passage through the sphere, the ray approximation of the scattered field is given by:

$$
\begin{array}{lrl}
\mathbf{u}_{p}^{s c}(r, z)=\hat{\mathbf{z}} e^{i \omega z / \alpha_{0}}\left(e^{i 2 \omega\left(1 / \alpha-1 / \alpha_{0}\right) R \cos j}-1\right) & r=R \sin j \leq R, & z \geq R \\
\mathbf{u}_{p}^{s c}(r, z)=\mathbf{0} & r>R
\end{array}
$$

Applying Hooke's law, it is readily shown that the scattered traction is simply proportional to the scattered displacements:

$$
\mathbf{t}_{p}^{s c}(r, z)=\frac{i\left(\lambda_{0}+2 \mu_{0}\right) \omega}{\alpha_{0}} \mathbf{u}_{p}^{s c}(r, z)
$$

We may now inject the ray approximation (3)-(4) in the representation theorem (1) to calculate the scattered wavefield in the far-field. Actually, we only need the amplitude of the $P$ wave scattered in the forward direction thanks to the optical theorem (Varatharajulu, 1977; Dassios et al., 1987; Margerin and Sato, 2011). Physically, this theorem expresses that the interference of the forwardscattered wave with the incident plane wave entails a reduction of the total amplitude in the far-field, thereby ensuring energy conservation. For a unit amplitude incident wave, the forward-scattered $P$-wave may be expressed in the far-field as:

$$
\mathbf{u}_{p}^{s}(r=0, z \hat{\mathbf{z}})=\hat{\mathbf{z}} \frac{f^{p}(\hat{\mathbf{z}}) e^{i \omega z / \alpha_{0}}}{z}
$$

where $f^{p}(\hat{\mathbf{z}})$ is the scattering amplitude in the forward direction. Mathematically, the optical 
theorem may be written as:

$$
\sigma_{p}=\frac{4 \pi \alpha_{0}}{\omega} \operatorname{Im} f^{p}(\hat{\mathbf{z}})
$$

where $\sigma_{p}$ is the $P$-wave scattering cross-section and Im denotes the imaginary part of a complex number. Using the expression of the free space Green's functions found in Aki and Richards (2002, p. 72) and after some straightforward algebra we obtain from Eq. (1) and (3) the following expression for the forward scattering amplitude:

$$
f^{p}(\hat{\mathbf{z}})=\frac{i \omega R^{2}}{2 \pi \alpha_{0}} \int_{0}^{2 \pi} d \varphi \int_{0}^{\pi / 2}\left(1-e^{i 2 \omega\left(1 / \alpha-1 / \alpha_{0}\right) R \cos j}\right) \sin j \cos j d j
$$

The symbol $\varphi$ denotes the azimuthal angle with respect to the incident direction and the reader is reminded that $j$ is the incidence angle of a given ray on the spherical surface. Formula (7) is valid in the limit $\omega R^{2} / \alpha_{0} z \rightarrow 0$, which allows us to neglect the phase difference between the scattered wavelets upon propagation from points $(x, y, R)$ of the surface $S$ to the detection point $(0,0, z)$ located in the far-field in the forward direction. Likewise, to lowest order in the small quantity $R / z$, the geometrical spreading and polarization vector of the scattered $P$ wavelets may be approximated by $1 / z$ and $\hat{\mathbf{z}}$, respectively.

Van de Hulst (1981) discusses the physical interpretation of formula (7) in details. The first term in the parenthesis is completely independent of the properties of the scatterer and quantifies the role of the waves that are diffracted by the object. This contribution also shows up in the problem of diffraction by screens in optics. Note that in this work the term "diffraction" is employed in the restrictive sense of "Fraunhofer" diffraction as defined in optics. Diffraction phenomena that go beyond our simple ray approximation do occur in the vicinity of the scatterer. For example, the sharp discontinuity of $u^{s c}$ postulated in Eq. (3) is presumably invalid in a region whose width scales with the wavelength, and which is located at the edge of the surface $S$. As a consequence, our treatment is appropriate at sufficiently high frequency only. The second term of the integrand of Eq. (7) represents the effect of the waves that are directly transmitted through the object in the forward direction. Eq. (7) shows that it is the interference of diffracted and transmitted waves that is responsible for the scattering attenuation. The integrals in Eq. (7) can be performed analytically 
and upon using the optical theorem (6), we find:

$$
\sigma_{p}=4 \pi R^{2}\left(\frac{1}{2}+\frac{1-\cos \delta \phi}{\delta \phi^{2}}-\frac{\sin \delta \phi}{\delta \phi}\right)
$$

with $\delta \phi=2 \omega\left(1 / \alpha-1 / \alpha_{0}\right) R$ is a phase shift due to the scatterer. Formula (8) was previously derived by Korneev and Johnson (1996) using the exact series solution of the problem. In the high-frequency limit $\omega \rightarrow \infty$, one finds the well-known result $\sigma \rightarrow 2 \pi R^{2}$, i.e., the scattering cross-section tends to twice the geometrical cross-section of the scatterer $\sigma_{g}$. From an energy conservation perspective, the factor 2 is explained by the following equal contributions: (1) the rays that are refracted upon crossing the object; (2) the rays that are diffracted by the disruption of the wavefront in the shadow of the scatterer (Van de Hulst, 1981). Under the weak contrast assumption, the result (8) may also be applied to $S$ waves provided some obvious changes are made (see below). The key point is that the polarization of the incident $S$ wave is barely affected by the transmission through the scatterer. It then suffices to replace the $P$ wave speeds with the $S$ wave speeds in the definition of the phase shift, yielding: $\delta \phi=2 \omega\left(1 / \beta-1 / \beta_{0}\right) R$. A verification of the applicability of formula (8) for $S$ wave is shown in Figure 2. After the non-dimensionalization $\omega \rightarrow \omega R / \alpha_{0}$ for $P$ waves, and $\omega \rightarrow \omega R / \beta_{0}$, the similarity between the frequency dependence of the scattering cross-section of $P$ and $S$ waves for velocity-type scatterers is striking.

\section{Impedance-type perturbations}

We now consider the problem of impedance type perturbations. Comparison of Figures 2 and 3 reveals that the level of scattering -at high-frequency- is much weaker for impedance-type than for velocity-type perturbations. Furthermore, it is clear from Figure 3 that the frequency dependence of the scattering cross-section is different for $P$ and $S$ waves. As shown by a Taylor series expansion, formula (8) predicts that the scattering cross-section should be equal to 0 in the case of impedancetype perturbations, which is clearly incorrect. To find a sensible result we must now take into account the change of amplitude of the waves that are transmitted through the scatterer. The modification of formula (7) is straightforward and one obtains:

$$
\sigma_{p}=4 \pi R^{2} \int_{0}^{\pi / 2}\left(1-T_{p}^{m \rightarrow s}(j) T_{p}^{s \rightarrow m}(j)\right) \sin j \cos j d j
$$


where $T_{p}$ is the displacement $P$ to $P$ transmission coefficient for plane waves as given by Aki and Richards (e.g. 2002). The superscript $m \rightarrow s$ indicates transmission from the matrix to the sphere (and conversely for $s \rightarrow m$ ). Figure 3 shows that Formula (9) gives a very good approximation of the exact result for $\omega R / \alpha_{0}>10$. Integral (9) is not easily computed analytically. However, a reasonable result is obtained by taking the acoustic approximation $T_{p p}^{m \rightarrow s} T_{p p}^{s \rightarrow m} \approx 4 \rho_{0} \rho /\left(\rho+\rho_{0}\right)^{2}$, which yields $\sigma_{p} \approx 2 \pi R^{2}\left(\rho-\rho_{0}\right)^{2} /\left(\rho+\rho_{0}\right)^{2}$. It is worth noting that for weak contrasts, the first term in the parenthesis of Eq. (9) is almost cancelled out by the second. Following our previous interpretation of formula (7), it means that diffraction effects no longer play a role in the scattering attenuation. Physically, this makes sense since the phase front remains perfectly planar upon crossing the scatterer. To confirm our interpretation, we may compute the scattering cross-section by removing, from the incident flux, the part of the energy that is directly transmitted in the forward direction (i.e., the un-scattered radiation). This yields:

$$
\left.\sigma_{p}=2 \pi R^{2} \int_{0}^{\pi / 2}\left(1-\left(T_{p}^{m \rightarrow s}(j) T_{p}^{s \rightarrow m}(j)\right)\right)^{2}\right) \sin j \cos j d j
$$

where the first term in the parenthesis represents the total flux intercepted by the scatterer and the second quantifies the fraction of the flux that remains in perfect coherence with the incident wave (and is therefore indistinguishable from it). For weak contrasts the formulas (9) and (10) agree remarkably well, a result that may be proven formally by a Taylor expansion.

The case of shear waves requires extra care (see Appendix A for computational details). Indeed, the reflection coefficients of $S H$ and $S V$ waves differ at the interface between two media exhibiting an impedance contrast. In the case of the ray depicted in Figure 1, the $S V$ (resp. $S H$ ) component vibrates in (resp. perpendicular to) the plane of the Figure. Attention must also be paid to the fact that $S$ to $P$ conversions make the transmission coefficient complex for $j>\arcsin \beta_{0} / \alpha_{0}$. Polarization effects yield the following modifications of formulas (9)-(10):

$$
\begin{aligned}
& \sigma_{s}=4 \pi R^{2} \operatorname{Re} \int_{0}^{\pi / 2}\left(1-\frac{T_{s v}^{m \rightarrow s}(j) T_{s v}^{s \rightarrow m}(j)}{2}-\frac{T_{s h}^{m \rightarrow s} T_{s h}^{s \rightarrow m}}{2}\right) \sin j \cos j d j \\
& \sigma_{s}=2 \pi R^{2} \int_{0}^{\pi / 2}\left(1-\frac{\left.\mid T_{s v}^{m \rightarrow s}(j) T_{s v}^{s \rightarrow m}(j)\right)\left.\right|^{2}}{2}-\frac{\left(T_{s h}^{m \rightarrow s} T_{s h}^{s \rightarrow m}\right)^{2}}{2}\right) \sin j \cos j d j
\end{aligned}
$$

where Re denotes the real part of the integral and $T_{s v}$ is the $S V$ to $S V$ transmission coefficient (and 
similarly for $\left.T_{s h}\right)$. The reader is referred to Appendix $\mathrm{A}$ for additional comments on the derivation. Formula (11) follows from the optical theorem while formula (12) provides an interpretation in terms of energy conservation. As previously noted in the case of $P$ waves: $(1)$ the diffraction term cancels out because the transmission coefficients are close to 1. (2) As a consequence, the scattered energy may be interpreted as the flux intercepted by the scatterer minus the fraction which is directly transmitted in the forward direction. A comparison between the asymptotic formula (11) and the series solution of the BVP shows good agreement between the two for $\omega R / \beta_{0}>10$.

\section{Discussion and conclusion}

In this section, we examine the conjecture made by Kawahara (2011) and Calvet and Margerin (2018) about the validity of the Born (or Rayleigh-Gans) approximation at high-frequency in the case of impedance-type perturbations. Loosely speaking, the Born approximation discretizes a finite object into elementary volumes of dimension much smaller than the wavelength. The contribution of each volume to the scattering amplitude is computed to first order in parameter heterogeneity and subsequently summed taking into account the phase difference between the secondary sources of scattered waves. The limitations of this approach have been largely discussed in the literature in the case of velocity-type perturbations. Van de Hulst (1981, P. 87) shows that in the Born approximation, the scattering cross-section is typically proportional to $\omega^{4} \alpha_{0}^{-4} R^{6}\left(\alpha / \alpha_{0}-1\right)^{2}$. Thus, at high frequency, the Born approximation predicts an increase of the scattered amplitude which is unrealistically large, eventually causing a divergence of the scattering cross-section in the limit $\omega \rightarrow \infty$. It is worth pointing out that the divergence of the scattering amplitude is concentrated around the forward direction and should become noticeable when the phase-shift $\left|\omega\left(1 / \alpha-1 / \alpha_{0}\right) R\right|$ is of order 1 (in the case of incident $P$ waves, say). Wu and Zheng (2014) show that consideration of higher-order terms of the Born series removes the singularity.

Using Eq. (11a)-(11d) and Appendix B of Margerin et al. (2000), we have computed numerically the scattering cross-section of $P$ and $S$ waves in the Born (Rayleigh-Gans) approximation for impedance type perturbations. This theory agrees within a few percents with the series solution of the BVP and does a nice job at explaining the oscillations of the scattering cross-section as a function of frequency. It is very likely that this success can be explained by the absence of 


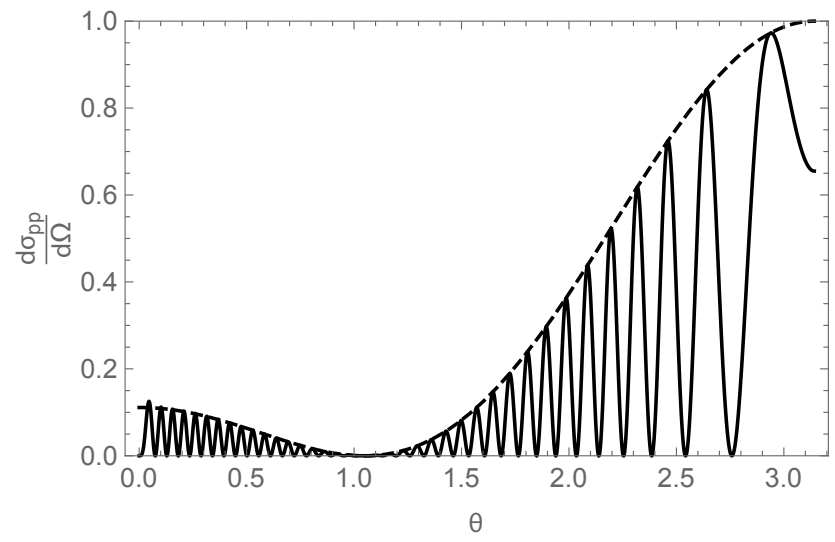

Figure 4: Differential scattering cross-section $d \sigma_{p p} / d \Omega$ as a function of scattering angle $\theta$ for $P$ to $P$ scattering in the case of $-5 \%$ impedance-type perturbations at non-dimensional frequency $\omega R / \alpha_{0}=60$. Note the rapid oscillations (solid line) inside the smooth envelope (dashed line).

phase-shift inside the velocity-type scatterer, as put forward by Kawahara (2011) and Calvet and Margerin (2018). It is also worth noting that in the Born approximation, the scattering amplitude can be factored into the product of a "shape factor" and a term which depends on the perturbation of the elastic tensor ( $\mathrm{Wu}$ and Aki, 1985b). Independently of the polarization of the incident and scattered wave, the latter is exactly zero in the forward direction in the case of impedance-type scatterer, which cuts-off any divergence of the scattering amplitude as suggested above. For this reason, Kawahara (2011) has suggested that the conversion scattering cross-section can be well approximated by the Born approximation at all frequencies, independent of the type of scatterers. Note, however, that the forward scattering amplitude can never be exactly zero because it is related to the extinction cross-section by the optical theorem and therefore assumes a finite value (possibly purely imaginary).

In Figure 3, we remark that there is a small but visible offset between the series solution and the Born approximation. This discrepancy can be traced back to the violation of the boundary conditions by the latter at the interface between the matrix and scatterer, i.e., the continuity of tractions is not ensured. As a consequence, the Born approximation predicts that the scattering cross-section is proportional to $\left(\rho-\rho_{0}\right)^{2} / \rho_{0}^{2}$, whereas ray theory strongly suggests a dependence on $\left(\rho-\rho_{0}\right)^{2} /\left(\rho+\rho_{0}\right)^{2}$. Indeed, if we (empirically) substitute the former with the latter in the Born approximation (see formulas below), the agreement with the series solution is greatly improved for $\omega R / c>10$ (where $c$ equals $\alpha_{0}$ or $\beta_{0}$ ). 
Having understood the limit of validity of the Born approximation, we may use it to exhibit the dependence of the scattering cross-section on the wavespeed ratio $\gamma=\beta_{0} / \alpha_{0}$. To do so, we remark that the scattering amplitude is a rapidly varying function of the scattering angle which oscillates inside a slowly-varying envelope. This property is illustrated in Figure 4 where we show the differential scattering cross-section for $P$ to $P$ scattering in the case of $-5 \%$ impedance-type perturbations at non-dimensional frequency $\omega R / \alpha_{0}=60$. Note that the differential scattering cross-section, which is proportional to the squared scattering amplitude, has been normalized by the maximum of its envelope. The oscillations are described with good accuracy with standard trigonometric functions of $\omega R / c$ so that we expect that the integral over $\theta$ is approximately equal to half the area delimited by the envelope and the horizontal axis. Using Appendix B of Margerin et al. (2000), the analytical formula for the envelope may be extracted by factoring out all the terms that depend on frequency that are therefore rapidly oscillating. In the case of $P$ to $P$ scattering, the slowly-varying envelope shown in Figure 4 is of the form: $\left(\rho-\rho_{0}\right)^{2}\left(\alpha_{0}^{2}-4 \beta_{0}^{2} \cos ^{2} \theta / 2\right)^{2} / \rho_{0}^{2}$. Integrating over the volume of the scatterer, we find:

$$
\sigma_{p p}=\frac{\pi R^{2}\left(\rho-\rho_{0}\right)^{2}}{2 \rho_{0}^{2}}\left(1-4 \gamma^{2}+\frac{16 \gamma^{4}}{3}\right)
$$

Proceeding similarly for $P$ to $S$ scattering conversions we find:

$$
\sigma_{p s}=\frac{\pi R^{2}\left(\rho-\rho_{0}\right)^{2}}{\rho_{0}^{2}}\left[\gamma\left(\frac{1}{3}-2 \gamma^{2}\right)+\frac{\left(1-\gamma^{2}+2 \gamma^{4}\right)}{2} \log \left(\frac{1+\gamma}{1-\gamma}\right)\right]
$$

and:

$$
\sigma_{s p}=\frac{\sigma_{p s}}{2 \gamma^{2}}
$$

where the last formula follows from reciprocity (e.g. Aki, 1992). In the case of $S$ to $S$ conversions, we find the particularly simple formula:

$$
\sigma_{s s}=\frac{5 \pi R^{2}\left(\rho-\rho_{0}\right)^{2}}{6 \rho_{0}^{2}}
$$

To summarize, we have computed the scattering cross-section of an impedance-type object at high frequency by three methods: the series solution of the BVP, an asymptotic method due to 
Van de Hulst (1981) based on ray theory, and the popular Born approximation. We found that the last two methods provide simple and relatively accurate approximations of the exact response. On the one hand, Born approximation describes well the oscillations of the scattering cross-section as a function of frequency and exhibits a small but systematic shift with respect to the exact solution. This slight discrepancy was explained by the violation of the boundary conditions at the surface of the scatterer. On the other hand, the ray approximation is in close agreement with the exact result but fails at predicting the oscillations of the scattering cross-section as a function of frequency. Our study confirms the conjecture of Kawahara (2011) and Calvet and Margerin (2018) that in the case of impedance-type scatterers, the Born approximation remains approximately valid at high frequency, in sharp contrast with the case of velocity-type scatterers. Perhaps the most interesting outcome of this short study is the clear demonstration that there is no geometrical regime for impedance-type scatterers, in the sense that their scattering response depends on their internal properties at all frequencies. Finally, we remark that results from Born approximation and ray theory may readily be extended to convex objects of otherwise arbitrary shape which may be useful to study the effect of shape preferred orientation on seismic attenuation (Calvet and Margerin, 2016).

\section{Data and Resources}

Figures were produced with the Mathematica software. The bibliography was produced with the aid of the style file bssa_usc.bst provided by P. Crotwell.

\section{Acknowledgments}

The comments of two anonymous referees helped improve the clarity of the manuscript.

\section{References}

Aki, K. (1992). Scattering conversions P to S versus S to P, Bull. Seismol. Soc. Am 82, 1969-1972.

Aki, K. and P. G. Richards (2002). Quantitative seismology. University Science Books.

Calvet, M. and L. Margerin (2012, Erratum 143, 139-141, 2018). Velocity and attenuation of scalar 
and elastic waves in random media: A spectral function approach, J. Acous. Soc. Am. 131, $1843-1862$.

Calvet, M. and L. Margerin (2016). Impact of grain shape on seismic attenuation and phase velocity in cubic polycrystalline materials, Wave Motion 65, 29-43.

Dassios, G., K. Kiriaki, and D. Polyzos (1987). On the scattering amplitudes for elastic waves, Zeitschrift für Angewandte Mathematik und Physik (ZAMP) 38, 856-873.

Foldy, L. L. (1945). The Multiple Scattering of Waves. I. General Theory of Isotropic Scattering by Randomly Distributed Scatterers, Physical Review 67, 107-119.

Kawahara, J. (2011). Scattering attenuation of elastic waves due to low-contrast inclusions, Wave Motion 48, 290-300.

Korneev, V. and L. Johnson (1996). Scattering of P and S waves by a spherically symmetric inclusion, Pure Appl. Geophys. 147, 675-718.

Margerin, L., M. Campillo, and B. Van Tiggelen (2000). Monte Carlo simulation of multiple scattering of elastic waves, J. Geophys. Res. 105, 7873-7892.

Margerin, L. and H. Sato (2011). Generalized optical theorems for the reconstruction of Green's function of an inhomogeneous elastic medium, J. Acous. Soc. Am. 130, 1-17.

Pao, Y.-H. and V. Varatharajulu (1976). Huygens' principle, radiation conditions, and integral formulas for the scattering of elastic waves, J. Acous. Soc. Am. 59, 1361-1371.

Sato, H. (1984). Attenuation and envelope formation of three-component seismograms of small local earthquakes in randomly inhomogeneous lithosphere, J. Geophys. Res. 89, 1221-1241.

Sato, H. (1989). Broadening of seismogram envelopes in the randomly inhomogeneous lithosphere based on the parabolic approximation: Southeastern Honshu, Japan, J. Geophys. Res. 94, 1773517747.

Stoffa, P., J. T. Fokkema, R. de Luna Freire, and W. Kessinger (1990). Split-step Fourier migration, Geophysics 55(4), 410-421. 
Torquato, S. (2002). Random Heterogeneous Materials: Microstructure and Macroscopic Properties. Springler-Verlag, New York.

Van de Hulst, H. C. (1981). Light scattering by small particles. New York: Dover Publications.

Varatharajulu, V. (1977). Reciprocity relations and forward amplitude theorems for elastic waves, J. Math. Phys. 18, 537-543.

Wu, R. and K. Aki (1985a). Elastic wave scattering by a random medium and the small-scale inhomogeneities in the lithosphere, J. Geophys. Res. 90, 10261-10273.

Wu, R. and K. Aki (1985b). Scattering characteristics of elastic waves by an elastic heterogeneity, Geophysics 50, 582-595.

Wu, R.-S. (1994). Wide-angle elastic wave one-way propagation in heterogeneous media and an elastic wave complex-screen method, Journal of Geophysical Research: Solid Earth 99(B1), $751-766$.

Wu, R.-S. and Y. Zheng (2014). Non-linear partial derivative and its De Wolf approximation for non-linear seismic inversion, Geophysical Journal International 196(3), 1827-1843. 
323 Ludovic Margerin^, Institut de Recherche en Astrophysique et Planétologie, Université Paul Sabatier 324 Toulouse III, C.N.R.S., C.N.E.S., 14 Avenue Edouard Belin, 31400 Toulouse France

325 ( ${ }^{\star}$ Corresponding author: ludovic.margerin@irap.omp.eu)

326 Marie Calvet, Institut de Recherche en Astrophysique et Planétologie, Université Paul Sabatier ${ }_{327}$ Toulouse III, C.N.R.S., C.N.E.S., 14 Avenue Edouard Belin, 31400 Toulouse France 


\section{List of Figure Captions}

Figure 1: Position of the problem. We consider a plane elastic ( $P$ or $S)$ wave incident on a sphere of radius $R$. The incidence angle of a given ray is denoted by $j$. The subscript 0 refers to the properties of the matrix with $\rho$ the density, $\alpha$ the $P$-wave velocity and $\beta$ the $S$-wave velocity.

Figure 2: Scattering cross-section (normalized by the geometric cross-section) of $P$ waves (a) and $S$ waves (b) as a function of non-dimensional frequency $k_{p, s} R$ for a velocity-type perturbation equal to $-5 \%$. Solid line: series solution of the BVP, dotted line: Ray theory.

Figure 3: Scattering cross-section (normalized by the geometric cross-section) of $P$ waves (a) and $S$ waves (b) as a function of non-dimensional frequency $k_{p, s} a$ for an impedance-type perturbation equal to $-5 \%$. Solid line: series solution of the BVP, dashed line: Born (Rayleigh-Gans) approximation, dotted line: ray theory.

Figure 4: Differential scattering cross-section $d \sigma_{p p} / d \Omega$ as a function of scattering angle $\theta$ for $P$ to $P$ scattering in the case of $-5 \%$ impedance-type perturbations at non-dimensional frequency $\omega R / \alpha_{0}=60$. Note the rapid oscillations (solid line) inside the smooth envelope (dashed line). 


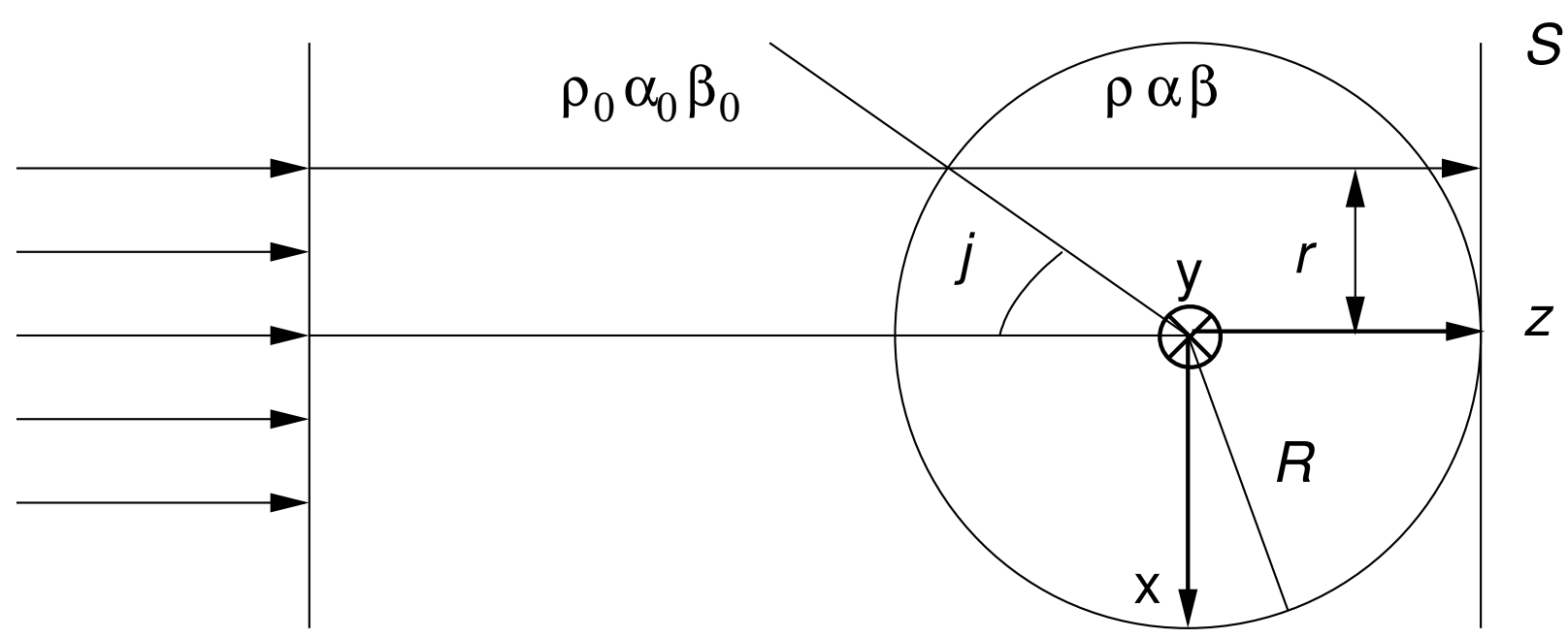

Figure 1: Position of the problem. We consider a plane elastic $(P$ or $S)$ wave incident on a sphere of radius $R$. The incidence angle of a given ray is denoted by $j$. The subscript 0 refers to the properties of the matrix with $\rho$ the density, $\alpha$ the $P$-wave velocity and $\beta$ the $S$-wave velocity.
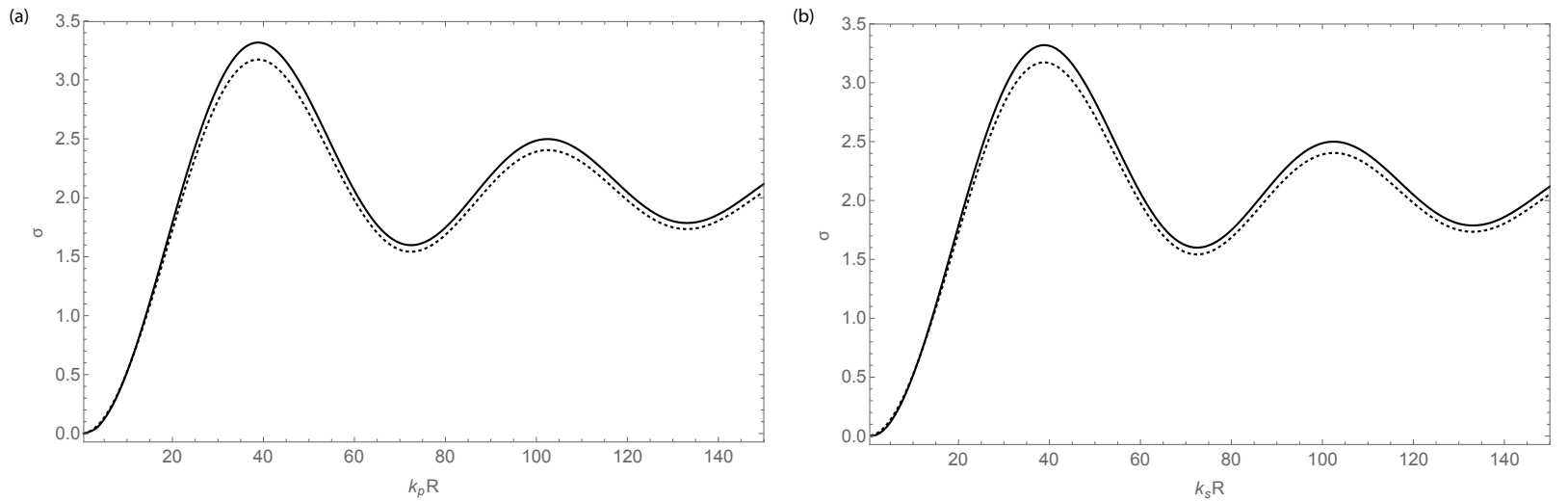

Figure 2: Scattering cross-section (normalized by the geometric cross-section) of $P$ waves (a) and $S$ waves (b) as a function of non-dimensional frequency $k_{p, s} R$ for a velocity-type perturbation equal to $-5 \%$. Solid line: series solution of the BVP, dotted line: Ray theory. 

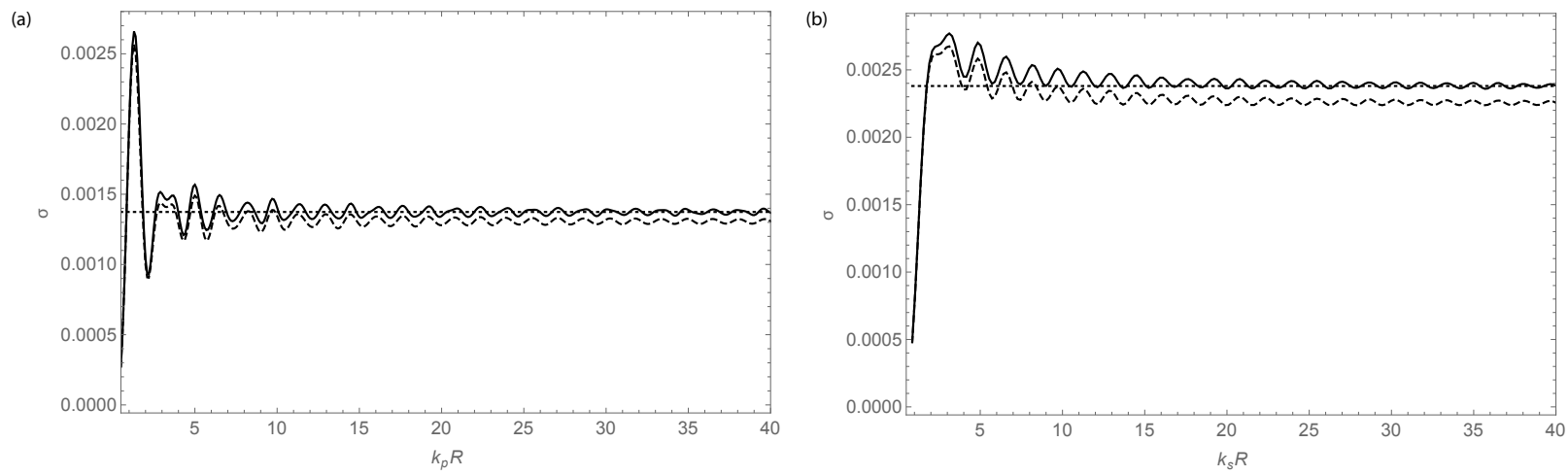

Figure 3: Scattering cross-section (normalized by the geometric cross-section) of $P$ waves (a) and $S$ waves (b) as a function of non-dimensional frequency $k_{p, s} a$ for an impedance-type perturbation equal to $-5 \%$. Solid line: series solution of the BVP, dashed line: Born (Rayleigh-Gans) approximation, dotted line: ray theory.

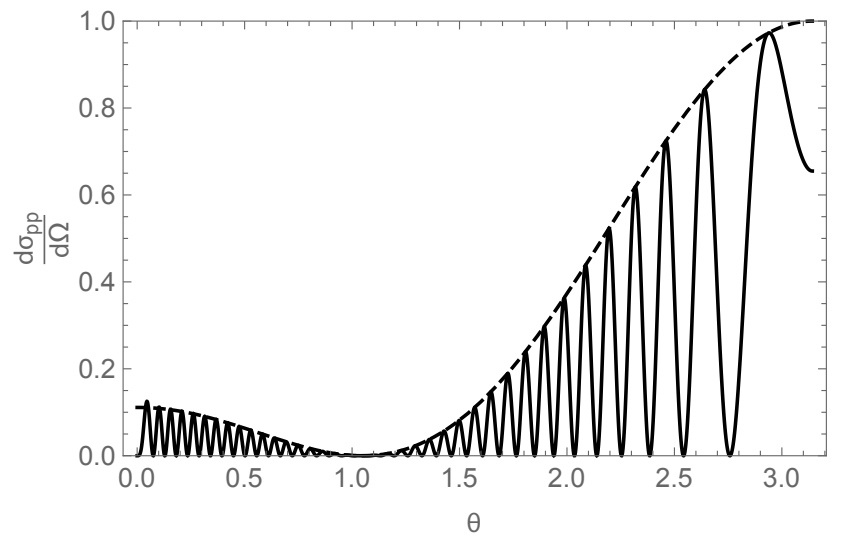

Figure 4: Differential scattering cross-section $d \sigma_{p p} / d \Omega$ as a function of scattering angle $\theta$ for $P$ to $P$ scattering in the case of $-5 \%$ impedance-type perturbations at non-dimensional frequency $\omega R / \alpha_{0}=60$. Note the rapid oscillations (solid line) inside the smooth envelope (dashed line). 
This appendix summarizes the main computational steps for the derivation of Eq. (11). Here, we consider the case of an incident unit amplitude $S$-wave polarized along the $x$ axis (see Figure 1). At the entrance point of the ray inside the sphere $(R \sin j \cos \varphi, R \sin j \sin \varphi,-R \cos j)$, we decompose the wave motion onto local $S V$ and $S H$ components. The reader may readily verify that an appropriate local basis is given by:

$$
\begin{aligned}
& \widehat{\mathbf{s h}}=\sin \varphi \hat{\mathbf{x}}-\cos \varphi \hat{\mathbf{y}} \\
& \widehat{\mathbf{s v}}=\cos \varphi \hat{\mathbf{x}}+\sin \varphi \hat{\mathbf{y}}
\end{aligned}
$$

Using this basis, the shear wavefield transmitted through the sphere may be written as:

$$
\mathbf{u}_{s}(x=r \cos \varphi, y=r \sin \varphi, z)=\left(A_{s v}(j) \cos \varphi \widehat{\mathbf{s v}}+A_{s h}(j) \sin \varphi \widehat{\mathbf{s h}}\right) e^{i \omega z / \beta_{0}}
$$

where $A_{s h, s v}$ are amplitude terms (possibly complex). Note that representation (A3) is valid for both velocity-type and impedance-type perturbations: only the interpretation of the amplitudes differs. In the case of velocity-type perturbations and neglecting the effect of transmission coefficients, $A_{s h, s v}$ are pure phase terms: $A_{s h}=A_{s v}=e^{i 2 \omega\left(1 / \beta-1 / \beta_{0}\right) R \cos j}$. In the case of impedance-type perturbations and using the notations of the main text, one has $A_{s h}=T_{s h}^{m \rightarrow s} T_{s h}^{s \rightarrow m}$ and $A_{s v}=T_{s v}^{m \rightarrow s}(j) T_{s v}^{s \rightarrow m}(i)$. The former amplitude term is real and independent of the incidence angle of the ray $j$, while the latter is possibly complex and depends explicitly on $j$. Subtracting the incident field from Eq. (A3) and reverting to the global cartesian basis, the scattered displacement and traction fields on the exit surface $S$ may be approximated as:

$$
\begin{aligned}
\mathbf{u}_{s}^{s c}(r \cos \varphi, r \sin \varphi, z)= & \left(A_{s v}(j) \cos ^{2} \varphi+A_{s h}(j) \sin ^{2} \varphi-1\right) e^{i \omega z / \beta_{0}} \hat{\mathbf{x}} \\
& +\left(A_{s v}(j)-A_{s h}(j)\right) \cos \varphi \sin \varphi e^{i \omega z / \beta_{0}} \hat{\mathbf{y}}
\end{aligned}
$$

$$
\mathbf{t}_{s}^{s c}(r \cos \varphi, r \sin \varphi, z)=i \mu_{0} \omega \mathbf{u}_{s}^{s c}(r \cos \varphi, r \sin \varphi, z) / \beta_{0}
$$

Eq. (A5) is straightforwardly deduced from Eq. (A4) by application of Hooke's law.

The other ingredients that are needed for the calculations are far-field approximations of the dis- 
placement and traction Green's functions in the far-field of the surface $S$. Consider a point $P(x, y, R)$ of $S$ and a detection point in the forward direction $D(0,0, z)$. The leading term of the transverse part of Green's tensor is given by:

$$
G_{n k}^{0}(D, P)=\frac{e^{i \omega(z-R) / \beta_{0}}}{4 \pi \rho_{0} \beta_{0}^{2} z}\left(\delta_{n k}-\hat{z}_{n} \hat{z}_{k}\right)
$$

As discussed in the body of the text, this approximation is valid only when the condition $z \gg \omega R^{2} / \beta_{0}$ is met for the phase. For the amplitude and polarization vectors, the much less stringent condition $R \ll|z|$ suffices. To the same level of approximations, the partial derivatives of the transverse part of $G$ with respect to the coordinates of the source point $P$ are given by:

$$
G_{n k, l}^{0}(D, P)=\frac{-i \omega e^{i \omega(z-R) / \beta_{0}}}{4 \pi \rho_{0} \beta_{0}^{3} z}\left(\delta_{n k}-\hat{z}_{n} \hat{z}_{k}\right) \hat{z}_{l}
$$

We are now in a position to calculate the traction term $\mathbf{n}^{\prime} \cdot \Sigma\left(\mathbf{x}, \mathbf{x}^{\prime}\right)$ that appears in Eq. (1). Applying Hooke's law and using component notation, we find:

$$
\hat{z}_{m} c_{m p k l}^{0} G_{n k, l}^{0}(D, P)=-i \mu_{0} \omega G_{n p}^{0}(D, P) / \beta_{0}
$$

Examination of Eq. (A4), (A5), (A6) and (A8) reveals that the two terms in the integrand of Eq. (7) are equal which yields:

$$
\begin{aligned}
& \mathbf{u}_{s}^{s c}(0,0, z \hat{\mathbf{z}})=\frac{-i \omega e^{i \omega z / \beta_{0}}}{2 \pi \beta_{0} z} \int_{0}^{2 \pi} d \varphi \int_{0}^{\pi / 2} d j {\left[\left(A_{s v}(j) \cos ^{2} \varphi+A_{s h}(j) \sin ^{2} \varphi-1\right) \hat{\mathbf{x}}\right.} \\
&\left.+\left(A_{s v}(j)-A_{s h}(j)\right) \cos \varphi \sin \varphi \hat{\mathbf{y}}\right]
\end{aligned}
$$

The integrals over $\varphi$ are easy to perform and yield the scattered shear wave in the forward direction:

$$
\mathbf{u}_{s}^{s c}(0,0, z \hat{\mathbf{z}})=\frac{i \omega e^{i \omega z / \beta_{0}}}{z \beta_{0}} \int_{0}^{\pi / 2}\left(1-A_{s v}(j) / 2-A_{s h}(j) / 2\right) d j \hat{\mathbf{x}}
$$

\section{Note that the $\hat{\mathbf{y}}$ component of the scattered field vanishes as required by symmetry considerations.} Upon substitution of $A_{s v, s h}(j)$ with $T_{s v, s h}^{m \rightarrow s}(j) T_{s v, s h}^{s \rightarrow m}(i)$ and application of the optical theorem, the result (11) follows. 